\title{
EL CAMPO EN LA RUMANÍA DEL SIGLO XXI: CAMPESINOS RELEGADOS ENTRE PRODUCCIONES DE MERCADO
}

\author{
José Díaz-Diego \\ Universidad Católica de Temuco. Chile \\ jose.diaz@uct.cl
}

\section{RESUMEN}

Durante la primera década del siglo XXI, la agricultura rumana ha profundizado en la dualización de su estructura socioproductiva. El agro rumano ha pasado con rapidez de ser un sector planificado a una agricultura de mercado caracterizada por un pequeño número de grandes explotaciones y un importante volumen de campesinos con pequeñas parcelas y escasos recursos económicos y tecnológicos. El artículo que sigue describe y analiza el contexto socioagrario del campo rumano, prestando especial atención a la región de Moldavia y a la posición relegada del campesinado, para concluir apuntando que la apertura neoliberal de la agricultura rumana ha generado un importante número de campesinos desprotegidos ante los designios del mercado y sobre los que recae, además, el prejuicio de ser desconfiados e inmovilistas.

Palabras clave: Rumanía, agricultura, campesinado, siglo XXI.

\section{ABSTRACT}

The Romanian agriculture has deepened into the bifurcation of its socio-productive structure during the first decade of the 21th century. From a planned agriculture, the sector has moved speedily to a market agriculture characterized by a small number of large farms versus a high volume of peasants with short plots and few economic and technical resources. The text describes and analyzes the socio-agrarian context of the Romanian countryside,

Fecha de recepción: septiembre 2013.

Fecha de aceptación: junio 2014. 
especially focused on the Moldova region and the relegated position of the peasantry, to concludes that the neoliberal openness of the Romanian agricultural sector has created an important number of market-unprotected peasants who also suffer the prejudices of distrust and immobility.

Keywords: Romania, agriculture, peasantry, $21^{\text {th }}$ century.

\section{INTRODUCCIÓN}

Tras la caída del régimen de Nicolae Ceauşescu en diciembre de 1989, el Frente de Salvación Nacional, dirigido por Ion Iliescu, comenzó a desarticular la agricultura comunal de Rumanía en enero de 1990, apenas un mes después de la revolución decembrista y antes de las primeras elecciones presidenciales celebradas tras la dictadura, el 20 de mayo de 1990. Estos prolegómenos de la reforma agraria, promulgada en 1991, apuntalaron la política agrícola y rural que los distintos gobiernos rumanos acometerían durante toda la última década del siglo XX, dirigida por líneas maestras como el desmantelamiento del sistema comunal y asociativo, la privatización y restitución del suelo y la subsunción del campo rumano en las lógicas neoliberales de mercado (Abrahams, 1996; Lerman, 1999; Meurs, 1999; Cartwright, 1999, 2000 y 2001; Verdery, 1999 y 2003; Aligica y Dabu, 2003; Luca, 2007 y 2010; Dropu, 2007; Hatos, 2007; Díaz-Diego, 2013a). Como consecuencia de ello, el sector agrícola de la Rumanía del siglo XXI ha visto cómo en el campo se agrandan las diferencias socioeconómicas y productivas entre la agricultura campesina, presente en la práctica totalidad del país, buena parte de la cual de subsistencia, y las grandes explotaciones agrarias con sus producciones volcadas al mercado, localizadas mayoritariamente en el sur de Crişana, en el Banaţ y en la cuenca baja de la fértil llanura danubiana.

Con el objetivo de analizar, describir y reflexionar sobre las razones y consecuencias socioagrarias de esta marcada y creciente dualización del sector agrícola rumano y siguiendo, entre otras, las consideraciones de Creswell (et al. 2003, 2004 y 2007), Onwegbuzie (et al. 2006 y 2007) y Discroll (et al. 2007) en cuanto a las ventajas de las metodologías complejas para abordar el estudio de los fenómenos socioculturales, para la realización de este trabajo se ha apostado por la articulación de una metodología mixta integrando las perspectivas cuantitativa y cualitativa a través de la combinación de métodos y técnicas estadísticas, etnográficas y de análisis geográfico regional, trabajando para ello la recopilación, análisis y triangulación de datos estadísticos y georreferenciados, procedentes principalmente del Instituto Nacional de Estadística de Rumanía, así como de datos etnográficos procedentes de la observación participante y las entrevistas abiertas realizadas a agricultores, periodistas, técnicos y políticos rumanos durante los distintos trabajos de campo realizados en Rumanía y en España desde 2008 a 2012. Cabe mencionar además que el presente artículo es resultado de un trabajo de mayor envergadura para la elaboración de la tesis doctoral del autor sobre las reformas agrarias de Rumanía en el que, además del estudio de una amplia bibliografía especializada en campesinado y reformas agrarias de Europa del Este, se aplicaron técnicas documentales, archivísticas y etnohistóricas para el 
análisis de materiales procedentes tanto de numerosos fondos y archivos rumanos, como de otros países europeos (Díaz-Diego, 2013b). El texto que sigue está sustentado también por dicho trabajo.

\section{DEL CONTEXTO AGRÍCOLA RUMANO}

La agricultura y el medio rural han jugado y juegan un papel fundamental en la historia de Rumanía. La agricultura y la ganadería han marcado el rumbo social y económico del país carpático desde sus inicios como principado medieval hasta la actualidad. El sector agrario participa hoy de la estructura económica del país como uno de los sectores más representativos del PIB nacional, con un valor de producción superior a los 14.438 millones de euros anuales (INS, 2014), y del desarrollo del bienestar social de los rumanos, especialmente de los que habitan en entornos rurales, es decir, 9.256 .000 personas, el $46 \%$ de la población total de Rumanía (INS, 2013). Además, prácticamente la mitad de la población que vive en el medio rural trabaja directamente en la agricultura, según los datos oficiales, si bien este porcentaje ha de ser bastante más elevado debido a la dificultad de registrar rigurosamente el trabajo familiar no declarado que, al cabo, es el que sostiene a buena parte de las pequeñas explotaciones agrarias ${ }^{1}$, lo que es tanto como decir, a la gran mayoría de las economías familiares rurales.

Los retos de la agricultura rumana son numerosos y no del todo sencillos. Por poner sólo algunos ejemplos, pueden citarse la falta de cooperación y mejora del capital social rural (Sandu, 2003), la falta de iniciativas locales y la excesiva fe depositada en las soluciones foráneas (Frunza y Voicu, 2003), el reducido tamaño de las explotaciones agrarias y de sus parcelas (Hirschhausen-Leclerc, 1994 y Mihailescu, 1997), el mayor peso de los lazos de parentesco que los objetivos económicos en el arrendamiento de tierras (Hatos, 2004), el importante volumen migratorio de población rural hacia entornos urbanos nacionales y/o países extranjeros (Constantinescu, 2003; Bleahu, 2004a; Sandu, 2004; Voicu, 2004), la persistencia de explotaciones agrarias familiares en cuyo seno el mismo origen de las familias (unas rurales y otras urbanas) genera intereses contrapuestos (Mihailescu, 1997 y 2006), el escaso desarrollo de actividades rurales no agrarias (Bleahu, 2003 y 2004b) o el alto riesgo de pobreza asociada con la explotación agraria familiar (Chirca y Tesliuc, 1999, y Tesliuc, Pop y Tesliuc, 2001, Alboiu, 2009), entre otros.

A lo anterior deben sumarse además otras dificultades añadidas como las deficitarias infraestructuras de comunicación y energía, el envejecimiento de la población, el aislamiento

1 Por delimitar y hacer más operativa la categoría, por pequeña explotación agraria entenderemos aquí, tal y como se expone más avanzado el texto, la finca rústica en explotación cuya reducida extensión no permite a sus propietarios, arrendatarios o tomadores generar una producción o renta que supere en mucho las propias necesidades básicas de la unidad familiar. En términos jurídico-administrativos y para el caso rumano, estas explotaciones son la amplísima mayoría de las inscritas en el catastro y los servicios de registro de la propiedad como «explotaciones agrícolas individuales sin titularidad jurídica». Hay que señalar, eso sí, que pequeña propiedad no es sinónimo automático de marginación social, hecho que atiende a otros planos socioculturales, pero sí de una significativa relegación económica y política en el conjunto del Estado pues, como se analiza a continuación, las actuales coordenadas socioeconómicas de la mayoría de los pequeños propietarios o arrendatarios agrícolas rumanos, escasamente cooperativizados, carecen de una efectiva capacidad de intervención en la planificación agraria y en los mercados de su sector. 
de muchas aldeas, las dificultades de escolarización secundaria y asistencia sanitaria especializada, la escasez de servicios, el incremento estructural de la pobreza, la dificultad de acceso al crédito por parte del pequeño campesino (Swinnen y Gow, 1997; Sandu, 1999; Tesliuc, Pop y Tesliuc, 2001; Voicu y Pop, 2002; Dan, 2004, Voicu, 2004 y 2005; Díaz-Diego y Márquez-Domínguez, 2009 y 2011), etc., lo que desincentiva la fijación de población joven, que percibe en el mundo urbano un lugar con mejores oportunidades.

Aún siendo parte de la Unión Europea, la agricultura rumana posee una estructura y un perfil productivo más próximos a los que poseían sus socios occidentales a principio de los años 60, que al que presenta actualmente el conjunto de la Unión Europea (Otiman, 2008). El rendimiento económico de la agricultura rumana se sitúa entre los 300 y $350 € /$ ha, 2,5 veces menos que sus vecinos occidentales ${ }^{2}$, cuyos campos rinden entre 750 y $800 € / \mathrm{ha}$. El Valor Añadido Bruto del agro rumano gira en torno a los $880-900 € /$ ha mientras que el del entorno europeo occidental asciende hasta los 1.950-2.000 €/ha, 2,2 - 2,5 veces más. El fuerte perfil autárquico y de semi-subsistencia de la mayor parte de explotaciones agrícolas de Rumanía hace que las familias campesinas autoconsuman aproximadamente el $50 \%$ de lo que producen, mientras que este porcentaje en la Europa occidental se reduce hasta el $10-20 \%$, según estadísticas oficiales. No obstante, autores como Cornelia Alboiu llegan a cifrar el autoconsumo campesino en el país en el 76\% de la producción familiar, al contrario que las explotaciones agrícolas de carácter más empresarial, que destinan al mercado más del 63\% de media de su producción (Alboiu, 2009). Sea como fuere, la autarquía del campesinado rumano y su desconexión con el mercado en cuanto a venta se refiere quedan meridianamente claras.

Otro factor que aleja el perfil de la agricultura rumana de los estándares de la Europa occidental es la irrigación. Tras la caída del régimen socialista en 1989, el colapso sociopolítico primero y la restitución de las tierras después, propiciaron no sólo una rápida y radical mutación de la estructura de la propiedad sino el desmantelamiento de las estructuras productivas y sus recursos tecnológicos, entre ellos, los canales y útiles de riego. En la actualidad, Rumanía no ha conseguido rehabilitar mucho más allá del 35\% de la red de canales que irrigaban los campos rumanos en 1989. Si bien este problema llega a parecer menor si se tiene en cuenta que aproximadamente el $50 \%$ de los hogares rurales del país aún no cuentan con canalización de agua potable.

Evidentemente, la falta de riego hace que la producción agrícola rumana dependa especialmente del agua de lluvia, factor desequilibrante que se complica más aún por la obsolescencia de la maquinaria agrícola -cuando la hay- y la masiva utilización de fuerza de tiro animal en detrimento de tractores y segadoras, difíciles de ver en proporciones comparables con las de la Europa occidental. Ello es especialmente llamativo en áreas tradicionalmente más pobres como en Moldavia. Lo anterior hace que la diferencia entre los máximos y los mínimos interanuales del conjunto de la producción oscilen de media unos $2.000 \mathrm{~kg} / \mathrm{ha}$ mientras que en la Europa occidental, la horquilla se reduzca hasta los $1.300 \mathrm{~kg} / \mathrm{ha}$ (INS, 2012a y 2012b).

2 Nos referimos al conjunto de países que conforman la Unión Europea occidental o anterior a la ampliación hacia el Este, iniciada en 2004. Dichos países son Alemania, Austria, Bélgica, Dinamarca, España, Finlandia, Francia, Holanda, Grecia, Irlanda, Italia, Luxemburgo, Portugal, Reino Unido y Suecia. 
Este complejo cúmulo de factores, entre los que destaca el insuficiente uso y gestión de los recursos ambientales y humanos que posee el país, ha desembocado en un sistema agrícola desequilibrado, con grandes desigualdades en relación con la propiedad de la tierra, el tamaño de las explotaciones y la práctica incapacidad de competir que las explotaciones campesinas poseen frente a las grandes explotaciones agroindustriales ni en superficie, ni en producción, ni en precios, ni en rendimiento, ni en tecnología, ni en acceso a insumos, ni en conocimiento, etc.

Ello, evidentemente, ha generado un sistema agroalimentario dual en el que las pequeñas explotaciones tienen escasísima capacidad para negociar los precios de sus insumos, sus necesidades o sus producciones, mientras que las grandes productoras apenas sí se encuentran con un único problema serio, el del acceso a la tierra, si bien restringido a áreas muy colapsadas, de importante tensión urbanística y un PIB elevado, como las proximidades de Bucarest (Pieniadz et al., 2009).

«Los productores agrícolas individuales tienen un poder económico muy limitado, que no les permite influir de manera decisiva ni en las condiciones de compra de los factores de producción, ni en la venta de sus productos agrícolas, ni en nada que influya a su favor en las condiciones de compra-venta de los productos agrícolas. El mercado de los productos agrícolas está dominado por un número reducido de compradores y sociedades comerciales que sí pueden adquirir productos, maquinaria, etc., influyendo a su favor en los precios de esos productos». Extracto de entrevista a Ilie Sârbu. Ex Ministro de Agricultura y ex Presidente del Senado de Rumanía.

Ahora bien, en el actual contexto de crisis económica hay que decir que las pequeñas explotaciones agrarias han soportado mejor el endurecimiento de las condiciones financieras al estar al margen de los mercados internacionales y prácticamente desconectadas de las finanzas nacionales, dada su autarquía y las dificultades que para el acceso al crédito han tenido desde la reconversión del país al libre mercado. No obstante, ello no es complaciente pues el nivel de subsistencia de buena parte de las familias campesinas que han capoteado mejor la crisis económica roza mínimos que, por escasos, quedan fuera de los intereses de los mercados y son difícilmente alienables.

«Los precios han subido y eso se nota, pero para el campo se compra poco, casi no hay dinero, y no ahora, hace mucho. La gente intenta usar todo el máximo tiempo posible. Las semillas son las de casa, el estiércol el de los animales de casa, lo que se recoge [cosecha] es lo que se come. Y esto era antes de la crisis, porque había poco y también ahora, que sigue habiendo muy poco. En los pueblos pequeños, la agricultura de familia no puede ser más pobre ya». Extracto de entrevista a Emil, campesino, 45 años. Călăraşi.

En el medio rural rumano la actividad agrícola está además prácticamente aislada de otras actividades económicas no agrarias, no pudiéndose beneficiar de las sinergias positivas que la presencia de otros sectores reportaría al dinamismo económico de la zona. En este sentido, 
y especialmente en áreas tradicionalmente desaventajadas, como el centro de Moldavia, el gobierno ha conseguido pocos avances en relación con la diversificación económica del medio rural, no tiene una estrategia claramente definida, y los técnicos y directivos dudan sobre las capacidades del campesinado para abanderar nuevas iniciativas económicas.

De media, el 64,2\% de la economía rural lo representa el sector primario, el 18,5\% el sector secundario y el $17,1 \%$ el sector terciario, si bien aquí están incluidas también las cifras correspondientes a los pueblos más grandes, algunos con actividad industrial y administrativa de relevancia. Ni incluso el turismo, con un floreciente desarrollo en los entornos rurales europeos, ha hecho acto de presencia de forma notable en buena parte del territorio rumano, con excepción de áreas montañosas como Apuşeni, Maramureş, Bucovina o Braşov además del Delta del Danubio. A nivel nacional, la capacidad del turismo rural oscila en torno a las 11.000 camas en unas 1.600 casas rurales.

\section{DE LAS EXPLOTACIONES Y EL SUELO AGRÍCOLA}

En cuanto al número de explotaciones agrícolas, en algo menos de una década se ha perdido el 14,02\% del total de ellas, pasando de las 4.485.000 que había en 2002 a las 3.856.000 en 2010, más del $99 \%$ explotaciones sin personalidad jurídica, es decir, explotaciones individuales de pequeño tamaño. Tan sólo han crecido las explotaciones agrícolas con tamaño y vocación comercial, que en 2002 eran unas 23.000 fincas y hoy representan unas 31.000 explotaciones (INS, 2012a).

Con el doble objetivo de frenar esta sangría de explotaciones campesinas que desaparecen, ralentizar el envejecimiento del campo incorporando jóvenes y liberar tierra que pudiera ser absorbida por otros agricultores, aumentando el tamaño de sus explotaciones y corrigiendo en lo posible la microfragmentación del parcelario, el gobierno rumano ha intentado en los últimos años convencer a los mayores con tierras en régimen de manos muertas, de que las vendan o arrienden. A través de las delegaciones provinciales del Ministerio de Agricultura, el gobierno ha ofrecido rentas vitalicias de hasta $50 €$ por hectárea arrendada y de hasta $100 €$ por hectárea vendida a precio de mercado, según directivos públicos entrevistados.

Las explotaciones agrícolas en Rumanía se dividen en explotaciones agrícolas sin personalidad jurídica y explotaciones agrícolas con personalidad jurídica. En el grupo de las explotaciones sin personalidad jurídica se encuentran las explotaciones individuales por un lado y las explotaciones de titularidad familiar por otro. Las primeras son el 99,07\% de las explotaciones rumanas y las segundas apenas alcanzan el 0,13\%. En adelante, al referirnos a explotaciones campesinas, estaremos haciendo referencia al conjunto de las explotaciones agrícolas individuales sin titularidad jurídica. En el segundo grupo, el de las explotaciones con personalidad jurídica, encontramos, en orden de importancia por su número, las sociedades comerciales con capital mayoritariamente privado $(0,43 \%$ del total $)$, las explotaciones propiedad de fundaciones, instituciones religiosas y centros educativos privados $(0,24 \%)$, las explotaciones propiedad de los ayuntamientos -municipalidades- y las juntas de distrito $(0,07 \%)$, las sociedades o asociaciones agrícolas $(0,04 \%)$, las explotaciones propiedad de otras instituciones públicas $(0,01 \%)$, las explotaciones propiedad de los institutos de educación secundaria, de las estaciones de experimentación agraria o de las unidades escolares de 
perfil agrícola $(0,005 \%)$, las sociedades comerciales con capital mayoritariamente público $(0,002 \%)$, las cooperativas $(0,002 \%)$ y las explotaciones gestionadas por trabajadores autónomos $(0,0001 \%)$ (tabla 1$)$.

De entre las explotaciones individuales, que son la mayoría del agro rumano, resulta además que de ellas, en torno al 80\%, son explotaciones agrícolas de perfil autárquico o de semisubsistencia, es decir, que la unidad familiar que las trabaja sólo cuenta con los ingresos económicos que se derivan de su pequeña propiedad, dedican la práctica totalidad de su jornada laboral al cultivo y manejo de la tierra a falta de un empleo más lucrativo, consumen la mayor parte de lo que producen y, con lo que sacan de ella, apenas les da para vivir. En términos socioeconómicos, ello supone literalmente vivir rozando el umbral de la pobreza, cuando no quedarse por debajo, y no tener la capacidad de acceder a prácticamente nada que no sea alimentarse y pagar pocas facturas.

Por otro lado, la superficie agrícola del país se ha mantenido más o menos constante, con ligeras pérdidas hasta 2007 y una leve recuperación de superficie a partir de 2008. En la actualidad, la superficie agrícola rumana tiene una extensión aproximada de 15.867.000 hectáreas, si bien repartidas de forma muy desigual entre las pequeñas y las grandes explotaciones. Por ejemplo, de esos algo más de 15,8 millones de hectáreas agrícolas, en torno a 8,5 millones pertenecen a las pequeñas explotaciones mientras que los restantes 7,4 millones de hectáreas son explotadas por las grandes fincas. Ello supone uno de los principales escollos de la agricultura rumana en lo tocante a la estructura de la propiedad y los niveles de producción y modernización, pues significa que la SAU media a disposición de una familia campesina no llega ni a las 2 hectáreas (1,95 ha por explotación) mientras que la SAU media de las explotaciones con entidad jurídica supera las 190 hectáreas.

Estos datos además pueden leerse en clave más social, como es la empleabilidad de las fincas. Y es que 8,5 millones de hectáreas de agricultura campesina ocupan a más de 7 millones de rumanos, es decir, cada hectárea tiene la virtualidad de emplear 0,83 personas, mientras que del otro lado, 7,4 millones de hectáreas de agricultura industrializada apenas ofrecen trabajo a 111.000 personas, lo que supone 0,001 personas por hectárea, 83 veces menos que la agricultura campesina.

Es cierto que el trabajo que ofrece la agricultura campesina es poco lucrativo y, que en buena parte de las ocasiones, se trata de un trabajo cuyos dividendos apenas consiguen sobrepasar el escalón de la autarquía. Hay que resaltar que si bien el salario medio del trabajador agrícola con nómina en Rumanía está en los $235 €$ al mes (INS, 2012a), en su cálculo entran las nóminas de todos los técnicos, ingenieros y gestores que emplea el sector agrario, con lo que el sueldo de los jornaleros es aún mucho menor que la media. Además, en un país con exiguas coberturas sociales y en un difícil contexto económico, el trabajo en una pequeña propiedad, aunque sea de semi-subsistencia, puede suponer la diferencia entre la pobreza y la miseria.

No debe dejarse atrás tampoco que existe un porcentaje no desdeñable del $7 \%$ de las explotaciones campesinas que han conseguido dar el salto cualitativo de dirigir sus producciones hacia el mercado, lo que significa que es una frontera traspasable y resulta posible mejorar las condiciones del campesinado a través de la mejor comercialización de sus productos. A ello hay que añadir que otro $1 \%$ de las explotaciones campesinas está intentando ese salto hacia el mercado y que la producción de otro $13 \%$ de las pequeñas explotaciones 


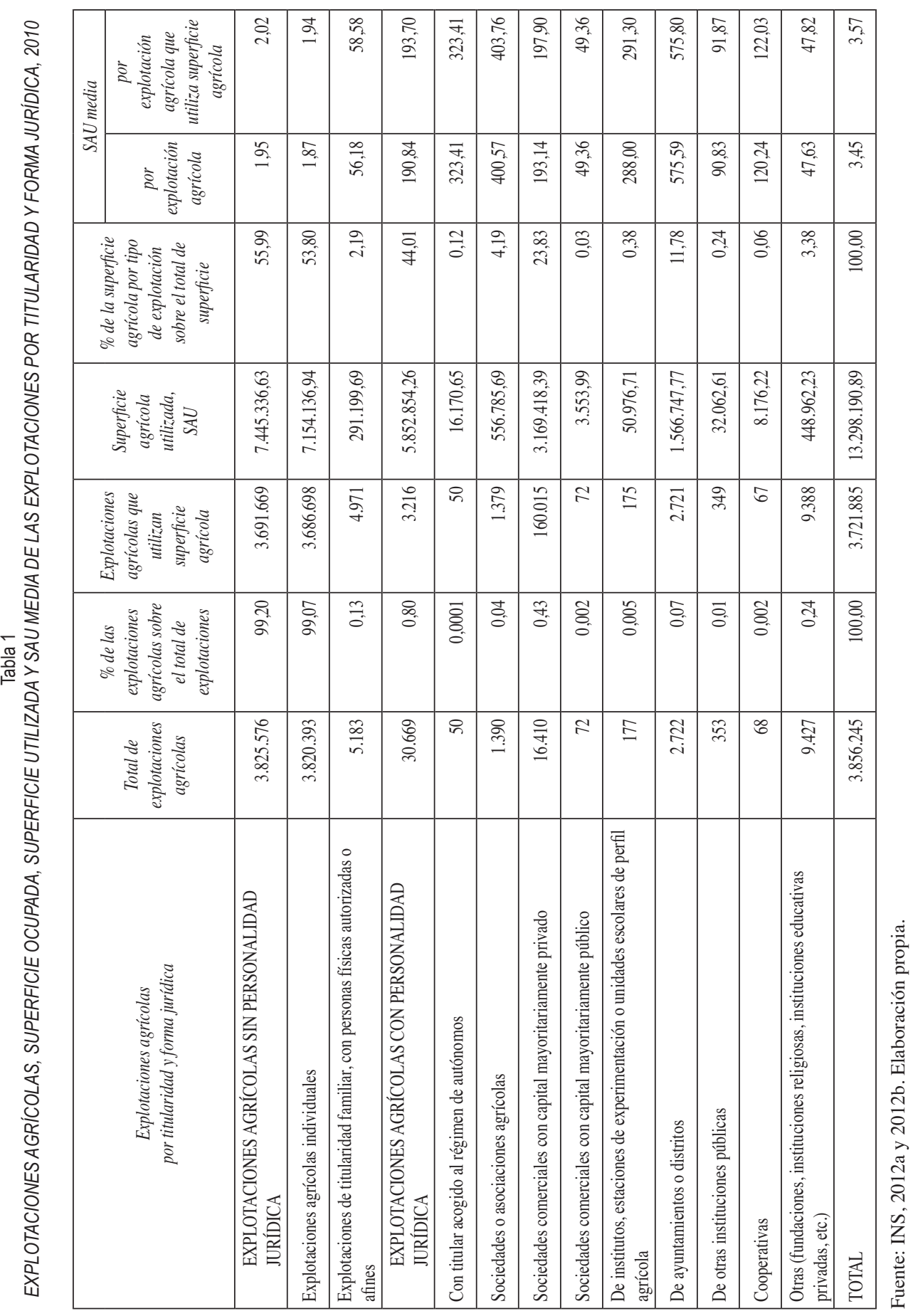


es un complemento de renta imprescindible para las economías familiares, al compatibilizar el cultivo a tiempo parcial de la parcela individual con otros trabajos fuera de la explotación campesina, con lo cual, la parcela de tierra de la familia campesina es una unidad económica aún fundamental en el engranaje del mundo rural, muy por encima, en términos sociales, de lo que supone la moderna agricultura.

Figura 1

EXPLOTACIONES CAMPESINAS VS. GRANDES EXPLOTACIONES, 2010
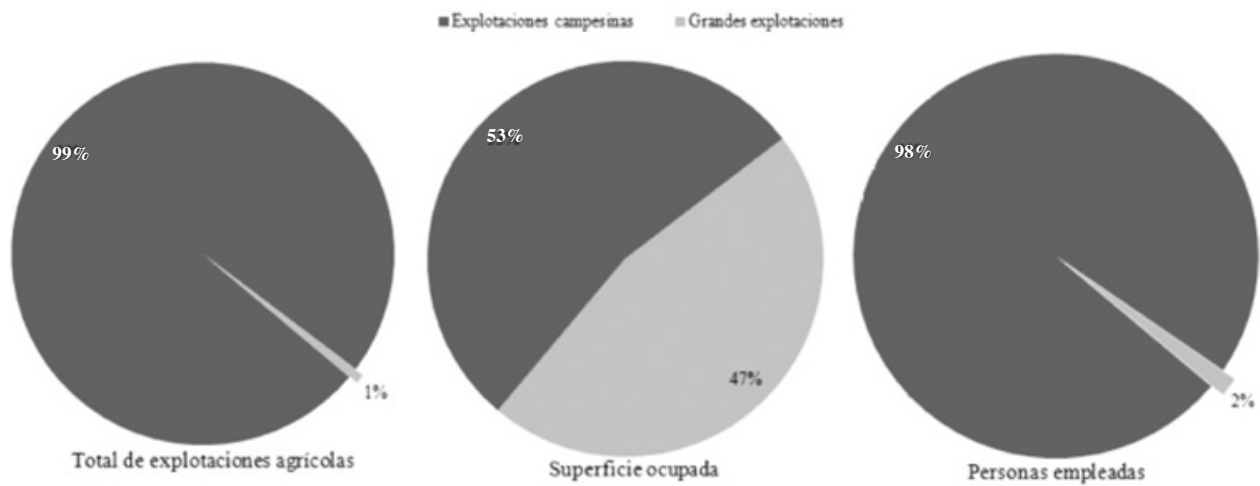

Fuente: INS, 2012a y 2012b. Elaboración propia.

En cuanto a los principales usos y aprovechamientos del suelo, el uso agrícola supone el $61,39 \%$ de la superficie rumana mientras que el suelo no agrícola ocupa el 38,61\% restante. Estos datos permiten observar que Rumanía es un país principalmente agrícola desde un punto de vista espacial. El suelo agrícola, que ocupa algo más de 14,6 millones de hectáreas, es principalmente arable. Los suelos arables ocupan más de 9,4 millones de hectáreas, lo que supone el $64,26 \%$ de la superficie agrícola y el $39,45 \%$ de la superficie total del país. El segundo aprovechamiento más importante del agro rumano son los pastos. Ocupan casi 3,2 millones de hectáreas, lo que supone el 22,47\% de la superficie agrícola y el 13,80\% del suelo rumano. En extensión le siguen los prados, con más de 1,5 millones hectáreas, es decir, el 10,45\% del suelo agrícola y el 6,42\% de la superficie nacional. Los viñedos siguen en importancia espacial a los prados, con 213.430 hectáreas $(1,46 \%$ del suelo agrícola y $0,90 \%$ del suelo rumano) y finalmente los frutales, que ocupan una extensión de casi 200.000 hectáreas $(1,36 \%$ de la extensión agrícola y $0,83 \%$ de la extensión nacional). Estos dos últimos aprovechamientos, viñedo y frutales, han sido de entre los usos agrícolas, los que mayor superficie han retrocedido en la última década: entre el año 2000 y el 2010 ha desaparecido el 21,61\% de la superficie ocupada por el viñedo y el $22,01 \%$ de la superficie planta de frutales. Por el contrario, la superficie arable se ha mantenido e incluso expandido un pequeño $0,25 \%$, en detrimento de los pastos, con una caída del 4,44\% que ha sido cultivado, abandonado asilvestrándose y/o construido 3 .

Desde el punto de vista del reparto territorial, los suelos arables se encuentran evidentemente en los distritos con tierras llanas y de elevado perfil productivo, como las localizadas en

3 Entre los años 2000 y 2010, el conjunto total del suelo urbano aumentó un 15,07\%. 
la gran cuenca del Danubio, la Meseta del Banat-Crişina y la Meseta Sur de Dobrogea. Son el caso de las tierras de Calaraşi, Ilfov, Ialomita, Girgiu, Teleorman, Braila, Olt, Constanza, Dolt, Galaţi o Tulcea, entre otros, además de áreas occidentales como Timiş, y otras igualmente aptas para el cultivo anual, como las de Botoşani o Vaslui, en Moldavia. Por el contrario, los prados y pastizales, recurso tradicional para el aprovechamiento de tierras de menor calidad, son proporcionalmente importantes en distritos con relieves más pronunciados, como Harghita, Maramureş, Hunedoara, Caraş-Severin, Bistrita-Nasaud, Valcea o Sibiu, entre otros.

Por su parte, los viñedos ocupan una superficie representativamente grande en las laderas de los Cárpatos y la Meseta Moldava, como en Vrancea, Galaţi, Vaslui, Buzau, Olt, Dolj, además de en las tierras llanas de Constanza, y los frutales, también aprovechando los desniveles carpáticos y las mesetas altas, se hacen presentes en Argeş, Gorj, Valcea, Dambovita, Prahova, Mehedinţi, Caraş-Severin, Buzau, Dolj y Timiş.

En el conjunto de los suelos no agrícolas sobresale por extensión la superficie forestal. Los bosques ocupan en Rumanía una extensión muy importante de terreno, especialmente en las áreas carpáticas. La foresta rumana se extiende por más de 6,7 millones de hectáreas, lo que supone prácticamente $1 / 3$ de la superficie del país. Las superficies inundadas también representan una extensión territorial importante, por encima de las 833.000 hectáreas $(3,50 \%$ de la superficie nacional). El suelo construido es el tercer uso en importancia por extensión entre los no agrícolas, con aproximadamente 730.000 hectáreas, al que le siguen el suelo degradado e improductivo (495.330 hectáreas) y finalmente el suelo ocupado por vías de comunicación (388.770 hectáreas).

Resulta especialmente destacable el retroceso en superficie del suelo improductivo y degradado, de un 22,13\% menos en 10 años, lo que está en parte relacionado con la necesidad del pequeño campesino de aprovechar al máximo la parcela de tierra restituida, incorporando a las estadísticas productivas los suelos de peor calidad que, en anteriores décadas, eran simplemente abandonados como eriales o tierras incultas. Tal ha sido el esfuerzo del campesinado por adecentar los suelos marginales que el saldo se ha vuelto positivo incluso cuando, como nos recuerda Mircea Bulgaru (2008a), el país sufre un acentuado proceso de deterioro de la calidad edáfica debido a la erosión, la acidificación, la salinización y la desertificación, especialmente en el sur y sureste rumanos, donde se localizan las tierras más fértiles.

«Cuando devolvieron las tierras, muchos se encontraron que la tierra no era buena porque había sido utilizada para otras cosas, porque había sido contaminada con basuras o porque te había tocado un monte de piedras que no era tuyo pero te había tocado y no servía para nada, ni para cultivos ni para crecer la hierba. Y los que no tenían otra cosa y tuvieron que trabajar la tierra, cultivaron incluso la tierra que no servía aunque estuviera mal y no valiese para nada». Extracto de entrevista a Mihai, campesino, 49 años. Vaslui.

\section{DE LOS RENDIMIENTOS Y LAS EXPECTATIVAS}

En cuanto a la producción, en torno al 70\% del PIB agrario lo genera el subsector vegetal, y dentro de éste, el $60 \%$ lo componen el cultivo y venta de cereales, oleaginosas, remolacha azucarera, patatas, hortalizas, legumbres, fruta y uva. La tendencia general de la superficie 
cultivada es decreciente. En los últimos 20 años ha retrocedido aproximadamente un 16,5\%, viéndose afectados cultivos fundamentales del agro rumano como el trigo, el viñedo o los frutales, al tiempo que otros han vivido un cierto periodo de expansión, como el ajo, la cebolla, la col, la avena, el girasol o la soja.

De igual forma, casi todas las producciones han jugado la misma suerte que la superficie cultivada, es decir, un retroceso de sus valores, excepto algunos cultivos explotados por la agricultura industrial, como el arroz, la avena o el girasol y aquellos otros que forman parte de la economía y la alimentación campesinas, como la col, el maíz, las patatas o los pimientos, lo que muestra la evidente dualidad de la agricultura rumana, con un sector industrial por un lado explotando nichos de mercado, y un sector campesino, por otro, preocupado por la perentoriedad de alimentarse a diario.

No obstante, también hay lugar para vías intermedias entre la producción industrial en extensivo y la producción familiar campesina, para cultivos como la ciruela o la fresa, que pueden suponer una alternativa de desarrollo y modernización para el pequeño agricultor, eso sí, si cuenta con la experiencia necesaria, el capital ineludible y la voluntad de asociarse, pues el tamaño sigue siendo un factor fundamental en el estratificado mercado agrícola rumano. En este sentido, de los rumanos que han trabajado fuera, y en concreto en las campañas agrícolas, como por ejemplo en la recogida de la fresa en Huelva (España), se espera y confía en que jueguen inteligentemente sus bazas aprovechando los fondos públicos que están a su disposición, aplicando su experiencia en el extranjero y aventurándose hacia la producción cooperativa, como sostienen directivos públicos entrevistados.

Si bien han retrocedido en importancia, los cereales siguen ocupando aproximadamente el $67 \%$ de la superficie total cultivada, especialmente el trigo y el maíz, aunque alcanzando rendimientos bastante bajos y desiguales inter-anualmente en comparación con la potencialidad de los suelos que los sostienen y las condiciones meteorológicas que los abrigan. Por ejemplo, del trigo se consigue una media de $3.660 \mathrm{~kg} /$ ha cuando se podría alcanzar los $5.500-7.000 \mathrm{~kg} / \mathrm{ha}$, y del maíz unos $4.000 \mathrm{~kg} / \mathrm{ha}$ cuando podrían llegarse a los $8.000 \mathrm{~kg} / \mathrm{ha}$. Evidentemente, la merma está relacionada con el desfase tecnológico y profesional debido a la descapitalización y a la insuficiente inversión pública y privada. Aun así, Rumanía es uno de los principales productores agrícolas de la Unión Europea y el segundo de Europa del Este tras Polonia, lo que evidencia su potencial y su capacidad de crecimiento.

Las lagunas comentadas hasta aquí influyen también en la productividad laboral, 4 veces inferior a la de Austria, 4,5 veces inferior a la de Alemania y 6 veces inferior a la de Francia, sin contar con la capacidad de producción de alimentos por agricultor, que en el caso rumano, llega a alimentar a 3 personas al año de media mientras que el agricultor medio europeo alimenta del orden de 20 a 30 personas en el mismo periodo (Bulgaru, 2008a). En términos más económicos, ello ha supuesto años de más de un $40 \%$ de la población activa produciendo menos de un $12 \%$ del PIB nacional y menos del 13\% de su VAB (Leonte, Giurca y Câmpeanu, 2002).

Si además a todo lo anterior se suma la escalada incesante de los precios, se obtiene una agricultura de una quebrada rentabilidad, al menos en términos de mercado (INS, 2012a y 2012b), pues en el fondo, la estrategia sobre desarrollo rural fijada en las conferencias de Cork (1996) y Salzburgo (2006) para la Unión Europea y los países entonces candidatos, ha sido claramente esquivada, especialmente en lo que se refiere a la corrección de las desigualdades entre el medio rural y el medio urbano, a través de una convergencia integrada, con la diversi- 
ficación económica como base, así como priorizando la sostenibilidad ambiental, la necesaria subsidiaridad pública, la simplificación de las gestiones, una seria planificación territorial a medio/largo plazo, la agilización de las inversiones económicas de rápido impacto sobre la población y el territorio, el viraje hacia políticas de gestión activa y participativa del desarrollo y una constante evaluación del proceso para corregir desviaciones y contingencias.

En iguales términos podríamos valorar el objetivo de la PAC (Política Agraria Común de la Unión Europea) de convertir al medio rural en algo más que un granero difícilmente rentable en el actual mercado globalizado, o lo que también ha venido conociéndose como refuerzo de la multifuncionalidad del medio rural, pues escaso ha sido el soporte público al campesino rumano, su protección económica y el impulso de su diversificación, el mantenimiento de vías simples de financiación, la aprobación de políticas para el reforzamiento de sus niveles de vida, la estimulación de sus actividades, la incentivación a la mejora de su producción por ejemplo con su reconversión hacia la producción ecológica de pequeña escala, la orientación de su labor hacia la organización horizontal y vertical para acceder al mercado, y la preservación de los mínimos necesarios para una vida digna en el campo; todo ello, señalado en el segundo pilar de la PAC, sobre desarrollo rural.

Para este gran objetivo, Rumanía ha recibido importantes fondos de desarrollo de la Unión Europea, especialmente a través del FEADER (Fondo Europeo Agrícola de Desarrollo Rural), con líneas evidentemente marcadas desde Bruselas en lo que respecta al desarrollo de la pequeña empresa agrícola, al apoyo de sus beneficios ecológicos y sociales en el medio rural y finalmente, al esfuerzo de diversificar el medio rural para no hacerlo depender exclusivamente de las actividades agrarias, que después el gobierno rumano se ha encargado de adaptar a su Programa Nacional de Desarrollo Rural 2007-2013. Según información facilitada en entrevista por Ilie Sârbu, ex Ministro de Agricultura y ex Presidente del Senado de Rumanía, el país invirtió más de 8.800 millones de euros, financiado por el FEADER en un $80 \%$ del total.

Aun con esta disposición presupuestaria, se ha constatado una incapacidad del gobierno rumano, por una parte, y del campesinado, por otra, de acceder a buena parte de los fondos europeos reservados para su desarrollo, en la medida en que ni uno ni el otro, pero especialmente el campesinado, han contado con el capital necesario para afrontar el porcentaje de inversión propia requerido en los proyectos de desarrollo rural. Así lo ha defendido Marius Lazăr, ex Secretario de Estado para el Trabajo de Rumanía, en una entrevista concedida al autor en Bucarest en 2008. Otros expertos, como los periodistas económicos Claudiu Raus, del Ziarul de Iaşi, o Alexandru Herman, del Ziarul de Bacău, inciden, en este sentido, sobre la rígida burocracia de la Administración, las rivalidades de partido en los entes regionales y locales ${ }^{4}$, así como directamente la corrupción política.

\section{DE LOS CAMPESINOS, MUCHOS RELEGADOS}

La conversión agrícola rumana del sistema comunista al de mercado no sólo supuso la pérdida de puestos de trabajo sino la reconfiguración de las categorías laborales vinculadas al

4 Para un mayor detalle de la gestión de los fondos europeos para el desarrollo rural en Rumanía puede consultarse el texto de Moga, L.; Antohi, V. y Neculita, M., (2012), «Usage of the European funds for agricultural and countryside development in Romanian rural areas», European Journal of Interdisciplinary Studies, vol. 4, núm. 2, pp. 56-65. 
campo, pues mientras que a principios de la década de los 90, casi el 95\% de los trabajadores agrícolas eran empleados, bien de cooperativas, bien de fincas del Estado, tras la reconversión de las estructuras económicas del país incrementaron con rapidez otras formas de organización laboral, como los autónomos o los «trabajadores familiares», es decir, aquellos que sin contrato, ni salario, ni propiedad directa de los medios, ocupan la mayor parte de su jornada laboral en la pequeña explotación familiar, en pos de la autarquía campesina, estrategia aún imprescindible para la precaria economía de muchas familias rurales. De forma claramente subestimada, pues se trata de cifras difícilmente registrables e incluso evitables -porque su declaración podría suponer perder prestaciones sociales-, el Instituto Nacional de Estadística de Rumanía cifró en torno a 40.000 los trabajadores agrícolas que podrían pertenecer a la categoría de empleados familiares sin contrato ni salario, pero que sin embargo no considera desempleados (INS, 2006). Sin duda, es una cifra en realidad mucho mayor.

En este sentido, la diferencia entre asalariados y no asalariados en el sector agrícola es aplastante, tanto en el periodo de expansión económica, aproximadamente hasta el año 2001, como de ahí en adelante con la contracción laboral del sector. En los últimos años, la diferencia entre el volumen de asalariados y no asalariados ha ido reduciéndose si bien no responde a un mayor número de asalariados, que ha permanecido constante a lo largo de los últimos 15 años, sino al descenso continuado de no asalariados o lo que es lo mismo, a la destrucción de explotaciones agrícolas. El volumen de asalariados permanece constante pues representan el número de empleados de las grandes explotaciones agrícolas, un sector que, si bien ha experimentado una cierto crecimiento en la última década, las labores mecanizadas han reducido sus necesidades de mano de obra, mientras que el sector de los no asalariados, pequeños propietarios más del $98 \%$ de ellos, son los responsables de su explotación y, en muchos casos, sin relevo generacional, su envejecimiento, además de la precariedad económica de la pequeña explotación, dibuja la caída en el número de ocupados en la agricultura, es decir, de la desaparición de explotaciones familiares.

Evidentemente, una de las razones de peso por la cual las explotaciones agrarias tienen un grave problema de relevo generacional son los dividendos. No hay cifras oficiales que permitan calcular los beneficios aproximados de la pequeña explotación sin que la producción de las grandes explotaciones distorsione los resultados, si bien los salarios percibidos en la agricultura y en comparación con otros sectores, pueden ser un indicador orientativo de gran valor para estimar la precariedad de la economía campesina. Hay que señalar aquí que el sector agrícola es uno de los peor pagados de la economía rumana. Un empleado agrícola gana, como se ha apuntado más arriba, una media de $234,55 €$ al mes, mientras que un transportista viene cobrando unos $352,04 € /$ mes, un minero unos $584 € /$ mes y finalmente, un empleado de banca unos 740,60 €/mes (INS, 2012b).

En su conjunto, todos ellos son sueldos bajos, pero los agrícolas son especialmente pírricos. No resulta muy complejo comprender las dificultades económicas a las que se enfrenta una familia campesina con ingresos de en torno a 235 euros al mes cuando, por ejemplo, 1 kilo de pan viene costando del orden de 0,61 euros, 6 chuletas de cerdo 4 euros, una botella de vino blanco de 0,75 L 3,37 euros, 1 kilo de harina 0,62 euros, 1 kilo de azúcar cuesta 1 euro, un tarro de tomate frito de 720 gr. vale 1 euro, 1 litro de aceite de girasol cuesta 1,3 euros, el mismo litro de aceite pero de oliva asciende hasta los 4,13 euros, una pechuga de pollo cuesta 3 euros, un cartón de huevos 3 euros, 1 kilo de uvas 1,10 euros, 1 trucha 
1,10 euros o un filete de salmón 9 euros. Súmense al precio de productos como éstos, de la conocida cadena de alimentación Carrefour, el resto de productos básicos, la luz, el agua, el teléfono -si se tiene-, el resto de impuestos, la ropa, la educación de los hijos, etc.

Vinculados a los bajos ingresos, sobresale entre las dificultades de las familias campesinas rumanas la escasa tierra y la rudimentaria tecnología a la que para trabajar han tenido acceso. Tanto en el periodo socialista como tras la caída del régimen de Ceauşescu y la restitución de las propiedades rústicas en 1991, la agricultura ha seguido siendo uno de los pilares fundamentales de la economía y de la vida de buena parte de la población rumana si bien el reparto de tierras generó una situación muy compleja por la microfragmentación del parcelario, acompañada de una dilatada crisis económica que desdibujó el perfil productivista de la agricultura rumana de la década de los 80 , retrotrayéndose en avances, como por ejemplo los de riego, mecanización, tecnología vegetal o zootecnia en lo que ha sido una auténtica «campesinización» del agro rumano.

Investigador: ¿Cuáles han sido los cambios más importantes de la agricultura de Iaşi en los últimos 10 años? Neculai: Después de 1990, después de la implementación de los fondos funciarios ${ }^{5}$, se ha asistido a una división excesiva de las superficies. Hemos pasado de una agricultura modernizada a una agricultura [con explotaciones de entre] 0,3 hectáreas y 10 hectáreas. (...) Menos a veces de 0,3 hectáreas. Aunque son excepciones, hemos tenido casos en que se llegó a dividir 1,8 hectáreas en 6 parcelas. Hemos tenido parcelas de hasta $18 \mathrm{~m}^{2}$, por eso hablo del problema de la tierra. (...) Después de la aparición de la legislación del dominio ${ }^{6}$ hemos pasado de una agricultura moderna a una agricultura campesina. Extracto de entrevista a Neculai Olariu. Director Adjunto de la Delegación Regional del Ministerio de Agricultura en Iaşi. // [Con la reforma agraria de 1991] «lo que se deseó fue que los activos de la agricultura pasaran a ser de propiedad privada, de tal manera que el sector privado aumentó su peso en más de un $93 \%$. Aún con ese elevado peso de lo privado, durante los primeros años después de la revolución, la producción agrícola registró caídas inferiores a las registradas en la industria, y durante los últimos años [la producción agrícola] se ha venido acercando al nivel de producción de 1989. (...) La agricultura rumana se acerca a la agricultura europea aunque las diferencias aún son muy significativas. Hoy en día la producción agrícola ha aumentado un $41 \%$ en comparación con el año 2000, mientras que en el periodo 1997-2000 había caído un 13,6\%. Este año [2008], la producción agrícola superará la de 1989 en un 3\%. (...) Los pequeños productores, que son hoy la mayoría, desarrollan su actividad en un ambiente que es poco favorable para una economía competitiva». Extracto de entrevista a Ilie Sârbu. Ex Ministro de Agricultura y ex Presidente del Senado de Rumanía.

Causa y consecuencia de lo anterior es que la mecanización del campo rumano, especialmente en lo que toca al uso de tractores y cosechadoras, es tres veces inferior al de la

5 Se refiere a la Ley de Tierras de 1991, en rumano Legea Fondului Funciar.

6 Igual que la nota anterior. 
Unión Europea. Buena parte de la maquinaria empleada está desfasada, mucha de ella aún de sello socialista. El campesinado rumano está fuertemente descapitalizado, sin acceso no sólo a la maquinaria sino, en muchas ocasiones, al simple consejo técnico. Todo lo anterior, incluida la superficie media de la explotación, la titularidad de la misma, la gente que la trabaja, los productos que cultivan y la rentabilidad de la finca, arroja un mapa de perfiles agrícolas en el que domina la producción campesina en la mayor parte del territorio rumano (figura 2).

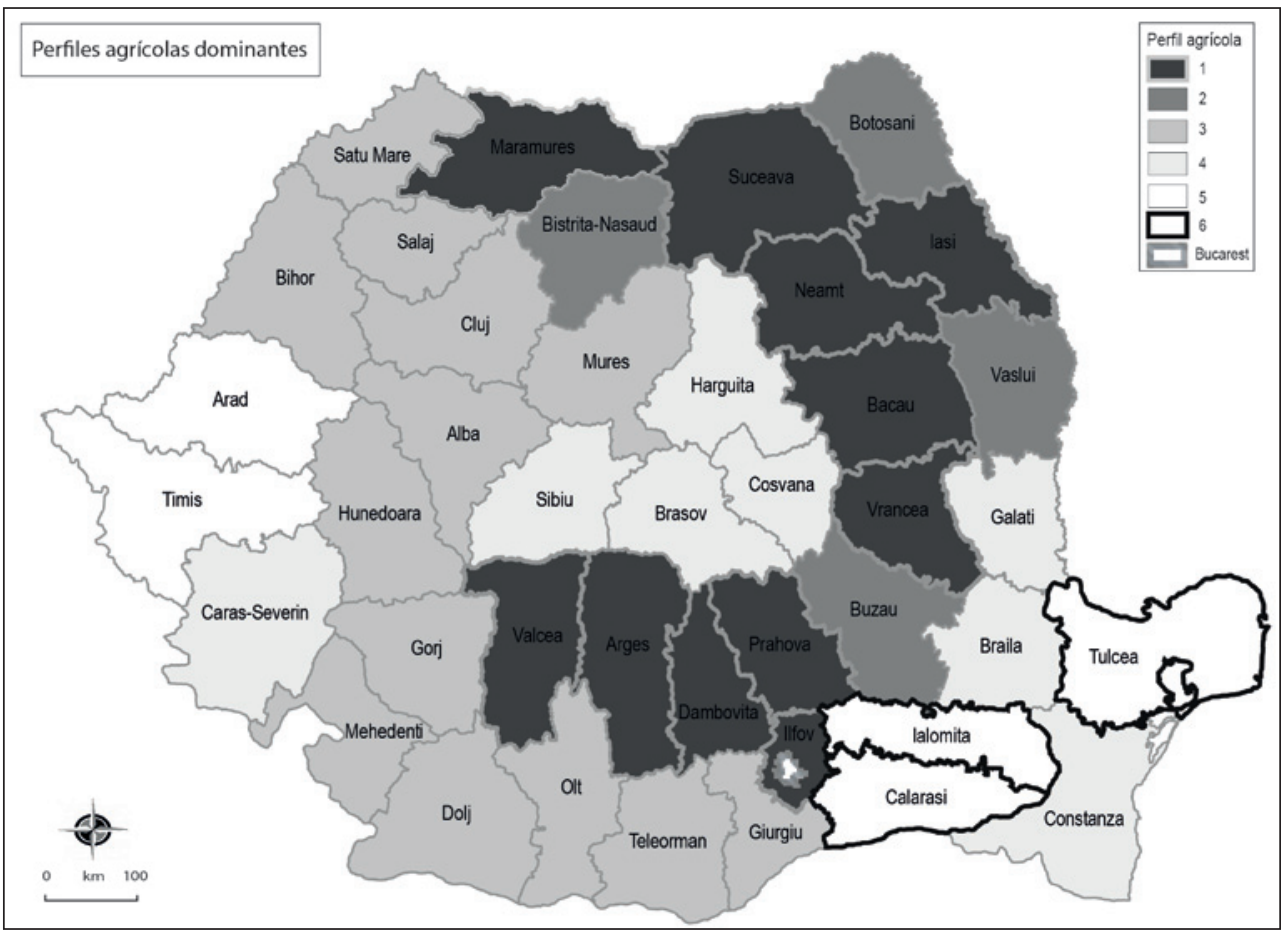

Perfiles agrícolas: (1) Agricultura campesina caracterizada por explotaciones agrarias familiares de pequeño tamaño, microfragmentadas, de importante carácter autárquico y escasamente mecanizada. (2) Agricultura campesina desarrollada en explotaciones de carácter familiar pero de mayor tamaño, con mayor excedente productivo e incipientemente mecanizadas. (3) Agricultura heterogénea dominada por la pequeña explotación de titularidad individual en transición hacia perfiles productivos más comerciales, coexistiendo con explotaciones de tamaño medio-grande, de producción principalmente cerealista y fuertemente mecanizadas. (4) Agricultura heterogénea donde coexisten la agricultura familiar en transición hacia perfiles más mercantiles y las asociaciones de producción cerealista fuertemente mecanizadas. (5) Agricultura indefinida, diversa, donde coexisten grandes explotaciones agrícolas privadas, mecanizadas y de producción principalmente cerealista, con explotaciones de tamaño medio de vocación comercial y pequeñas explotaciones familiares especializadas en producciones mayoritariamente hortícolas dirigidas en buena parte hacia el mercado local. (6) Agricultura cerealista desarrollada en grandes explotaciones privadas y/o asociadas, de vanguardia en su mecanización y cuya producción se dirige tanto al mercado nacional como a la exportación internacional. Fuente: MDRL, 2006; UAICI, 2008; INS, 2012a y 2012 b. Elaboración propia. 
Para mayor información, resulta necesario mencionar además que los servicios técnicos agrícolas no sólo resultan caros para los magros bolsillos campesinos sino que son notoriamente escasos también. La precariedad económica del medio rural y sus manifiestas desventajas en cuanto a comodidad de vida en comparación con los entornos urbanos hace que apenas el $5 \%$ de los veterinarios e ingenieros agroforestales rumanos estén ocupados efectivamente en tareas productivas vinculadas con su formación, muchos de ellos urbanitas que vuelven a la ciudad cuando tienen posibilidad de ello en aras de mejorar sus condiciones sociolaborales. Hay que recordar en este sentido que apenas se cuenta con técnicos agrarios de origen rural, pues tan sólo entre el 2 y el 3\% de los jóvenes rurales acceden a la universidad, debido principalmente a las precarias rentas de sus familias (Bulgaru, 2008b), una cifra que es, a la postre, una fuerte dosis de realidad para valorar las dificultades económicas del campesinado rumano y las razones de la bajísima profesionalización de las actividades agrícolas.

Aún así, entre muchos técnicos de la empresa privada y directivos de la Administración pública vinculados con el agro aún pesa más el argumento del conservadurismo y la pasividad del campesinado a la hora de explicar su paupérrima situación que el de las difíciles condiciones socioeconómicas en las que se desarrolla su vida, proyectando una imagen del campesino que roza la de un sujeto incapaz de comprender el mundo que le rodea, desperdiciando voluntariamente las oportunidades de mejora que se le brindan, lo que deriva fácilmente hacia una cuestionable y superficial proposición de: «si no mejoran, es porque no quieren».

En el siguiente extracto de entrevista, Stefan Talamba, Director Ejecutivo de la Delegación del Ministerio de Agricultura en Bacău, expone los esfuerzos de su delegación por concienciar a los pequeños agricultores de su distrito, de que deben apostar por la búsqueda de nuevas iniciativas dirigidas al mercado y a la eficacia de la producción, siguiendo modelos como el de la Holanda posbélica, con el evidente riesgo anacrónico. En la entrevista, si bien expone líneas clásicas sobre modernización del campesinado, no se enfatizan, más bien se minimizan, los aprietos de la familia campesina para explotar su finca de forma tradicional, cuánto no más para dar el salto hacia la intensificación. Y aunque en el discurso consciente hace responsable al campesinado de su situación, llegando a tildarlos de borregos..., lo que se desprende de su explicación es justamente lo contrario, la desastrosa gestión pública del proceso de privatización y restitución de la propiedad, incorregible hasta hoy, en cuanto a la desorientación que produjo en los agricultores verse reconvertidos de empleados a campesinos prácticamente de la noche a la mañana, a su suerte, sin experiencia alguna ni en agricultura autogestionada ni en voluntades de los mercados, tremendamente cambiantes a la postre:

Investigador: ¿Cuál es el sector económico más importante en esta zona [Moldavia rumana]? Stefan: La agricultura. Investigador: ¿Podríamos hablar de la evolución de esta agricultura desde la caída del comunismo en el 89 hasta ahora? ¿Cuáles han sido los principales cambios en la agricultura? Stefan: Los principales cambios han venido de la mano de la propiedad. El $90 \%$ son propietarios de pequeñas propiedades, de 1,5 hectáreas de media. Investigamos su nivel de asociacionismo y es prácticamente cero, porque buena parte de ellos recelan que el período comunista pueda volver. Investigador: ¿Entonces, es un problema de 
mentalidad? Stefan: Por supuesto. (...) Hay quien recela de la vuelta del período comunista (...). [Los campesinos] no tienen capacidad para volverse realmente innovadores, no confian en los bancos, no confian en hacer negocios con gente de fuera... Investigador: Me ha dicho que las propiedades aquí son muy pequeñas, de en torno a 1,5 hectáreas. Stefan: Sí, pero nosotros les decimos que, aunque tengan poca tierra, que vengan con una idea. Siempre pueden arrendar después. Investigador: ¿Pero tienen ayudas para arrendar? Stefan: No, pero son rentas mиy baratas, muy baratas. Pero ellos no tienen espíritu emprendedor. No se han adaptado a las condiciones normales de la Unión Europea y de ahí el impacto de la emigración. Continúan con un espíritu anticuado. Tenemos que hablar con la gente que se queda y con la gente que ha estado en regiones más ricas de Europa, para compartir experiencias, que traigan ideas y que inviertan en proyectos agrícolas aquí. [Los campesinos] tienen mentalidad de empleados, no de empresarios. $Y$ eso es muy importante. Investigador: ¿Y en su opinión, qué deben hacer para cambiar esto? Stefan: Campañas de información. Tenemos aquí en el distrito de Bacău relaciones con una asociación holandesa, y un experto nos ayuda viniendo y hablando con la gente sobre la Unión Europea, sobre el futuro que puede ofrecer la agricultura. Él explica cómo en Holanda, cuando el Plan Marshall, con todo ese dinero americano entregado después de la II Guerra Mundial, la gente cogió aquel dinero para comprar vacas, aves, el campo se puso a trabajar de una forma más eficiente. Se modernizaron. Y necesitamos modelos. (...) Tenemos una línea de presupuesto para invertir en más campañas de información para hacer que la gente de las zonas rurales descubra las oportunidades de las nuevas actividades agrícolas. Creo que es muy tarde pero es el primer paso. Es el primer dinero que se invierte en un proyecto rural para hacer que la gente cambie su mente. Investigador: Pero Rumanía durante el periodo comunista fue uno de los más importantes productores agrícolas de Europa del Este. En aquella agricultura, los campesinos de ahora eran trabajadores intensivistas. Sabían qué significaba la producción intensiva, sabían qué eran los tractores, sabían qué era el riego mecanizado, conocían todo aquello.... Stefan: Sí, pero en primer lugar, a la gente se le cortaron sus raíces con la tierra. En el periodo comunista se les arrebataron sus tierras y se las organizaron. Había un jefe que le decía al resto de la gente: _ vamos a trabajar aquella tierra, mañana vamos todos a trabajar aquella otra. La gente no desarrolló un espíritu de la autosuperación, de la autodecisión. [Fueron] como ovejas. Pero después de dos generaciones, la gente que había labrado sus tierras había muerto. Y la gente que ahora tiene las tierras había sido como ovejas. No habían manejado antes sus propias tierras. Para ellos, sus propias tierras sin ayuda, sin tractores... no eran nada. Y día a día se fueron empobreciendo más y más. Investigador: Entonces esta generación ha necesitado aprender a manejar de nuevo sus tierras... Stefan: La gente obtuvo sus tierras de nuevo pero no sabían qué hacer con ellas porque eran trabajadores, no campesinos. En segundo lugar, en este largo período de privatización, nadie salvó los canales de riego, los tractores, las herramientas... Investigador: ¿Qué ocurrió con esas infraestructuras? Stefan: Se destruyeron. Desde la privatización no se ha sabido invertir ni admi- 
nistrar. Por otra parte, en Rumanía hemos cambiado [hacia] el mercado. Rumanía tiene muchas tierras sembradas de cereal pero no son eficientes. Durante los últimos 50 años [la producción cerealista se había venido destinando principalmente al mercado ruso], pero después de la revolución, nuestras relaciones de vecindad con los rusos se destruyeron y tuvimos que cambiar nuestros productos para adaptarnos al nuevo mercado, pero sin dinero, sin experiencia. Fueron cambios demasiado rápidos. Extracto de entrevista a Stefan Talamba. Director Ejecutivo de la Delegación del Ministerio de Agricultura en Bacău.

Visto lo anterior, no resulta especialmente complejo comprender la desconfianza que el campesinado siente hacia la Administración y hacia todo lo que no sea su propio esfuerzo, lo que a su vez desmitifica que el escaso asociacionismo presente en el agro esté únicamente vinculado con el pasado comunista y el temor a perder el control sobre la propiedad privada de sus tierras. Parece estarlo, y mucho, con 20 años acumulando frustraciones de promesas políticas y técnicas incumplidas, que ahondan en el recelo de lo común como vía de desarrollo y mejora, lo que finalmente impide el fortalecimiento del capital social.

En lo que respecta al asociacionismo agrícola, los distintos gobiernos rumanos no parecen haber estimulado convenientemente la idea de cooperación y economía social, lo que ha favorecido el incremento de tierras abandonadas por dueños o muy mayores o muy frustrados ante la incapacidad de ganarse dignamente la vida en el campo. Y cuando desde el mundo político llegan algunas voces convencidas de la problemática que ello supone, resulta que en vez de proponer políticas de estímulo para el cooperativismo, se pretende corregir el problema únicamente gravando a impuestos las tierras incultas, como si fuesen las grandes fincas y no las pequeñas explotaciones las que están dejando de cultivar sus tierras. Evidentemente, esta subida de tasas a las tierras en régimen de manos muertas no grava a las arcas de los terratenientes, sino a las escasos ahorros de los jubilados agrícolas y de los jóvenes que, pequeños propietarios, han abandonado la actividad agraria en pos de mejores condiciones laborales. A la postre, ello sólo beneficia a la gran propiedad, que puede adquirir a mejor precio las tierras que, obligados por los impuestos, venden las familias campesinas.

\section{CONSIDERACIONES FINALES}

Desde la restitución de la propiedad privada y el reparto de tierras en 1991, pero muy marcadamente durante la primera década del nuevo siglo, el sector agrario rumano ha experimentado una rápida campesinización de su estructura, tanto en lo que respecta al tamaño y manejo de las explotaciones, como en cuanto a las características de las familias que las trabajan. Paralelamente, un pequeño número de grandes fincas, especialmente localizadas en las áreas de mayor potencial agrario, como la cuenca baja del Danubio, se han mecanizado, tecnologizado y volcado hacia las producciones de mercado. Esta moderna y competitiva agricultura contrasta con una agricultura campesina, minoritaria durante la época comunista pero socialmente mayoritaria hoy, por ejemplo en regiones como la Moldavia rumana, cuya penetración en el mercado nacional es mínima, está fuertemente dirigida al autoconsumo y al mercado local, genera pocos dividendos y, aunque más resistente ante los vaivenes mercantiles, cubre con dificultad las necesidades económicas de los trabajadores de la tierra. Este 
desequilibrio convive además con un medio rural alejado aún de los estándares europeos en cuanto a infraestructuras, dotaciones y servicios públicos cuyas negativas consecuencias son leídas no pocas veces, y por parte de políticos y representantes de la Administración, como efectos de un irreal inmovilismo campesino en vez de como resultados de serias deficiencias en la gestión y planificación públicas del sector agrario.

\section{AGRADECIMIENTOS}

El presente trabajo ha contado con el apoyo del proyecto FONDECYT de Iniciación núm. 11130213, financiado por CONICYT Chile para el periodo 2013-2016, llevado a cabo desde el Departamento de Antropología de la Universidad Católica de Temuco (Chile), y el proyecto nacional de I+D GECOA ref. CSO2010-18764, para el periodo 2011-2013, llevado a cabo por el Instituto de Desarrollo Local de la Universidad de Huelva (España) y financiado por el Ministerio español de Economía y Competitividad. Finalmente, agradezco la valiosa dirección del trabajo por parte de Juan Antonio Márquez Domínguez, y las constructivas aportaciones de los evaluadores anónimos del Boletín de la Asociación de Geógrafos Españoles.

\section{REFERENCIAS}

ABRAHAMS, R. (1996): After socialism. Land reform and social change in Eastern Europe. Oxford. Berghahn Books.

ALBOIU, C. (2009): «Subsistence agriculture in Romania. A modus vivendi?». 111th EAAEIAAE Seminar 'Small Farms: decline or persistence» Papers. Canterbury. University of Kent.

ALGICA, P. y DABU,A. (2003). «Land reform and agricultural reform policies in Romania's transition to the market economy: overview and assessment». Eastern European Economics, vol. 41, n ${ }^{\circ} 5,49-69$.

BLEAHU, A. (2003): «Rural non-farm activities in two Romanian counties». The rural non-farm economy and livelihood diversification in Romania. Bucarest. University of Greenwich, DFID y Banco Mundial.

BLEAHU,A. (2004a): «Romanian migration to Spain. Motivation, networks and strategies», en New patterns of labour migration in Central and Eastern Europe (Pop, D., ed.). ClujNapoca. ENPO.

BLEAHU, A. (2004B): «O perspectiva istorica asupra sectorului ne-agricol din mediul rural: 1930-2002». Calitatea Vietii, $\mathrm{n}^{\circ} 2$.

BULGARU, M. (2008a): «The agriculture», en Knowing Romania. Member of the European Union (Vacarel, I.; Bulgaru, M.; Ionescu, C.; Mitrut, C. y Voineagu, V., coords.). Bucarest. Romanian Academy, Romanian National Institute of Statistics, Romanian Statistical Society y National Foundation of Arts and Sciences.

BULGARU, M. (2008b): «The population», en Knowing Romania. Member of the European Union (Vacarel, I.; Bulgaru, M.; Ionescu, C.; Mitrut, C. y Voineagu, V., coords.). Bucarest. Romanian Academy, Romanian National Institute of Statistics, Romanian Statistical Society y National Foundation of Arts and Sciences. 
CHIRCA, C. y TESLIUC, E. (1999): De la saracie la dezvoltare rurala. Bucarest, INS y Banco Mundial.

CARTWRIGHT, A. (1999): Implementating land reform in post-communist Romania. Tesis Doctoral. Warwick. Universidad de Warwick.

CARTWRIGHT, A. (2000): «Avoiding 'collectivisation': land reform in Romania 19901992». Working Paper, núm. 4. Halle. Max Planck Institute for Social Anthropology.

CARTWRIGHT, A. (2001): The return of the peasant. Land reform in post-Communist Romania. Aldershot. Ashgate.

CONSTANTINESCU, M. (2003): «International circular migration to Spain. A case study». Papers Sibiu, n $^{\circ}$ 18. Disponible en: www.iccv.ro/Nyess/Papers_Sibiu_2003/18.\%20 monica\%20constantinescu.pdf

CRESWELL, J.W. y PLANO CLARK, V.L. (2007): Designing and conducting mixed methods research. Thousand Oaks. Sage Publications.

CRESWELL, J.W., FETTERS, M. e IVANKOVA, N. (2004): «Designing a mixed methods study in primary care». Annals of the Family Medicine, vol. 2, n 7, 7-12.

CRESWELL, J.W., PLANO CLARK, V. L., GUTMANN, M. y HANSON, W. (2003): «Advanced mixed methods research designs», en Handbook of mixed methods in social and behavioral research (Thakkori, A. y Teddlie, C., eds.). Thousand Oaks. Sage Publications.

DAN, A. (Coord.) et Al. (2004): Diagnoza locuirii: lipsa unei locuinte si locuirea in conditii precare. Bucarest. Institutul de Cercetare a Calitatii Vietii.

DÍAZ-DIEGO, J. (2013a): «Preludios de la contrarreforma agraria en la Rumanía de 1990: la caída del régimen, el Frente de Salvación Nacional y la decapitación de la agroindustria colectiva». Actas de las VIII Jornadas Interdisciplinarias de Estudios Agrarios y Agroindustriales. Buenos Aires. Universidad de Buenos Aires.

DÍAZ-DIEGO, J. (2013b): Las reformas agrarias de Rumanía, 1864-1991. Tierra, agricultura y campesinado. Tesis Doctoral. Inédita. Huelva. Universidad de Huelva.

DÍAZ-DIEGO, J. y MÁRQUEZ DOMÍNGUEZ, J. A. (2009): «Unos se van, ¿y los que se quedan? Población mayor, trabajo y agricultura en Rumanía». Analele Ştiinţifice ale Universității Alexandru Ion Cuza din Iaşi - seria Geografie, $\mathrm{n}^{\circ}$ 54, 117-130.

DÍAZ-DIEGO, J. y MÁRQUEZ DOMÍNGUEZ, J. A. (2011): «Difficult aging in rural Romania: peasants, pensions and agriculture». Journal of Eurasian Studies, vol. 3, n 3 , 92-116.

DRISCOLL, D.L. et al. 2007. «Mersing qualitative and quantitative data in mixed methods research: how to and why not». Ecological and Environmental Anthropology, vol. 3, $\mathrm{n}^{\mathrm{o}}$ $1,19-28$.

DROPU, C. (2007): Agricultura si politicianismul. Un secol de politica agrara in Romania 1907-2007. Iaşi. Editura Sedcom Libris.

FRUNZA, M. y VOICU, B. (2003): «The rural non-farm economy: exit from poverty chance or illusion?». The rural non-farm economy and livelihood diversification in Romania. Bucarest. University of Greenwich, DFID y Banco Mundial.

HATOS, A. (2004): «Darea in parte a terenurilor in România: exploatare sau efectul pietelor nefunctionale». Sociologie Româneasca, vol. 2, n 2, 106-123.

HATOS, A. (2007): Economica politica a restructurarii si reformei agrare. Disponible en: http://www.scrigroup.com/afaceri/economie/Economia-politica-a-restructur61963.php 
HIRSCHHAUSEN-LECLERC, B. (1994): «L'invention de nouvelle campagnes en roumanie». L'Espace géographique, $\mathrm{n}^{\circ} 1$.

INS (Instituto Nacional de Estadística de Rumanía) (2006): Ancheta structurala in agricultura. Bucarest. Institutul National de Statistica.

INS (Instituto Nacional de Estadística de Rumanía) (2012a): Recensamantul general agricol, 2010. Bucarest. Secretariatul Tehnic Central. Institutul National de Statistica.

INS (Instituto Nacional de Estadística de Rumanía) (2012b): Baza de date TEMPO - serii de timp. Bucarest. Institutul National de Statistica.

INS (Instituto Nacional de Estadística de Rumanía) (2013): Recensamantul populatiei si locuintelor, 2011. Rezultate definitive. Bucarest. Institutul National de Statistica.

INS (Instituto Nacional de Estadística de Rumanía) (2014): Valoarea productiei ramurii agricole - serie noua, 2012. Bucarest. Institutul National de Statistica.

LEONTE, J., GIURCA, D. y CÂMPEANU, V. (2002): Romania agriculture and EU accession. Bucarest. European Institute of Romania.

LERMAN, Z. (1999): «Land reform and farm restructuring: what has been accomplished to date?». 111th Annual Meeting of the American Economic Association Papers, pp. 271-275.

LUCA, L. (2007): «Romania: large semi-subsistence farm sector, result of wrong strategic approach?». Budapest. 104th EAAE-IAAE Seminar 'Agricultural Economics and Transition' Papers.

LUCA, L. (2010): «A retrospect of land market liberalization in Romania». Agricultural Economics and Rural Development, vol. 7, $\mathrm{n}^{\circ}$ 2, 293-304.

MDRL (Ministerio de Desarrollo, Obra Pública y Vivienda de Rumanía) (2006): Atlas Teritorial al României. Instrument de Analiza Teritoriala. Bucarest. Ministerul Dezvoltării, Lucrărilor Publice şi Locuinţelor.

MEURS, W. (1999): «Land reform in Romania - A never-ending story». South East Europe Review, vol. 2, n $2,109-122$.

MIHAILESCU, V. (1997): «Doua sate in tranzitie». Revista de Cercetari Sociale, $\mathrm{n}^{\circ} 3$.

MIHAILESCU, V. (2006): «Institutional 'transition' and 'post-communist' changes in Romania», en Ten years of European Studies in Cluj-Napoca. Globalism, globality, globalisation (Gyémán, L.; Jucan, M. y Rotar, C., coords.). Cluj-Napoca. Presa Universitara Cluj-Napocaeana.

MOGA, L., ANTOHI, V. y NECULITA, M. (2012): «Usage of the European funds for agricultural and countryside development in Romanian rural areas». European Journal of Interdisciplinary Studies, vol. 4, $\mathrm{n}^{\circ} 2$.

ONWEGBUZIE, A.J. y COLLINS, K.M.T. (2007): «A typology of mixed methods sampling designs in social science research». The Qualitative Report, vol. 12, $\mathrm{n}^{\circ}$ 2, 281-316.

ONWEGBUZIE, A.J. y LEECH, N.L. (2006): «Linking research questions to mixed methods data analysis procedures». The Qualitative Research, vol. 11, n 3, 474-498.

OTIMAN, P. (2008): «Rural development and agriculture: opportunities to attenuate the economic and financial crisis and to resume economic growth». Agricultural Economics and Rural Development, vol. 5, $\mathrm{n}^{\mathrm{o}} 3-4,97-128$.

PIENIADZ, A. et al. (2009): «Small farmers in the Romanian dairy market: do they have a future?». 111th EAAE-IAAE Seminar 'Small Farms: decline or persistence» Papers. Canterbury. University of Kent. 
SANDU, D. (1999): Spatiul social al tranzitiei. Iasi. Polirom.

SANDU, D. (2003): Sociabilitatea in spatiul dezvoltarii. Incredere, toleranta si retele sociale. Iasi. Polirom.

SANDU, D. (2004): «Cultura si experienta de migratie in satele României». Sociologie Româneasca, vol. 2, n 3, 179-201.

SWINNEN, J. y GLOW, H. (1997): «Agricultural credit problems and policies during the transition to a market economy in Central and Eastern Europe». Policy Research Group Working Paper, $\mathrm{n}^{\circ} 6$.

TESLIUC, C., POP, L. y TESLIUC, E. (2001): Saracia si sistemul de protectie sociala. Iasi. Polirom.

UAICI (Universidad Alexandru Ioan Cuza de Iasi) (2008): Datele agrare 2002 si 2003. Información cedida por Ionel Muntele, profesor del Departamento de Geografía. Iasi. Universitatea Alexandru Ioan Cuza din Iași.

VERDERY, K. (1999): «Fuzzy property: rights, power and identity in Transylvania's decollectivization», en Uncertain transition. Ethnographies of change in the postsocialist world (Burawoy, M. y Verdery, K., eds.). Oxford. Rowman \& Littlefield Publications.

VERDERY, K. (2003): The vanishing hectare. Property and value in postsocialist Transylvania. Londres. Cornell University Press.

VOICU, B. (2004): Resurse, valori si stategii de viata. Spatii sociale de alegere in tranzitie. Tesis Doctoral. Bucarest. Universidad de Bucarest.

VOICU, B. y POP, L. (2002): «Pupil's performances», en Rural education in Romania. Conditions, challenges and strategies of development (Jigau, M., ed.). Bucarest. MarLink.

VOICU, M. et al. (2005): Romanian farms and farmers facing the EU regulations. Iasi. Editura Expert Projects. 